\title{
Addition of a fast GC to SIFT-MS for analyses of individual monoterpenes in mixtures
}

\section{Supplementary Material}

Michal Lacko ${ }^{1,2}$, Nijing Wang ${ }^{3}$, Kristýna Sovová ${ }^{\text {, Pavel Pásztor }}{ }^{1}$, Patrik Španě ${ }^{1}$

${ }^{1}$ The Czech Academy of Science, J. Heyrovský Institute of Physical Chemistry, Dolejškova 2155/3, 18223 Prague 8, Czech Republic

${ }^{2}$ Faculty of Mathematics and Physics, Charles University in Prague, Ke Karlovu 3, 12116 Prague, Czech Republic ${ }^{3}$ Air Chemistry Department, Max-Planck-Institut für Chemie, Hahn-Meitner-Weg 1, 55128 Mainz, Germany

Correspondence to: Michal Lacko (michal.lacko@jh-inst.cas.cz)

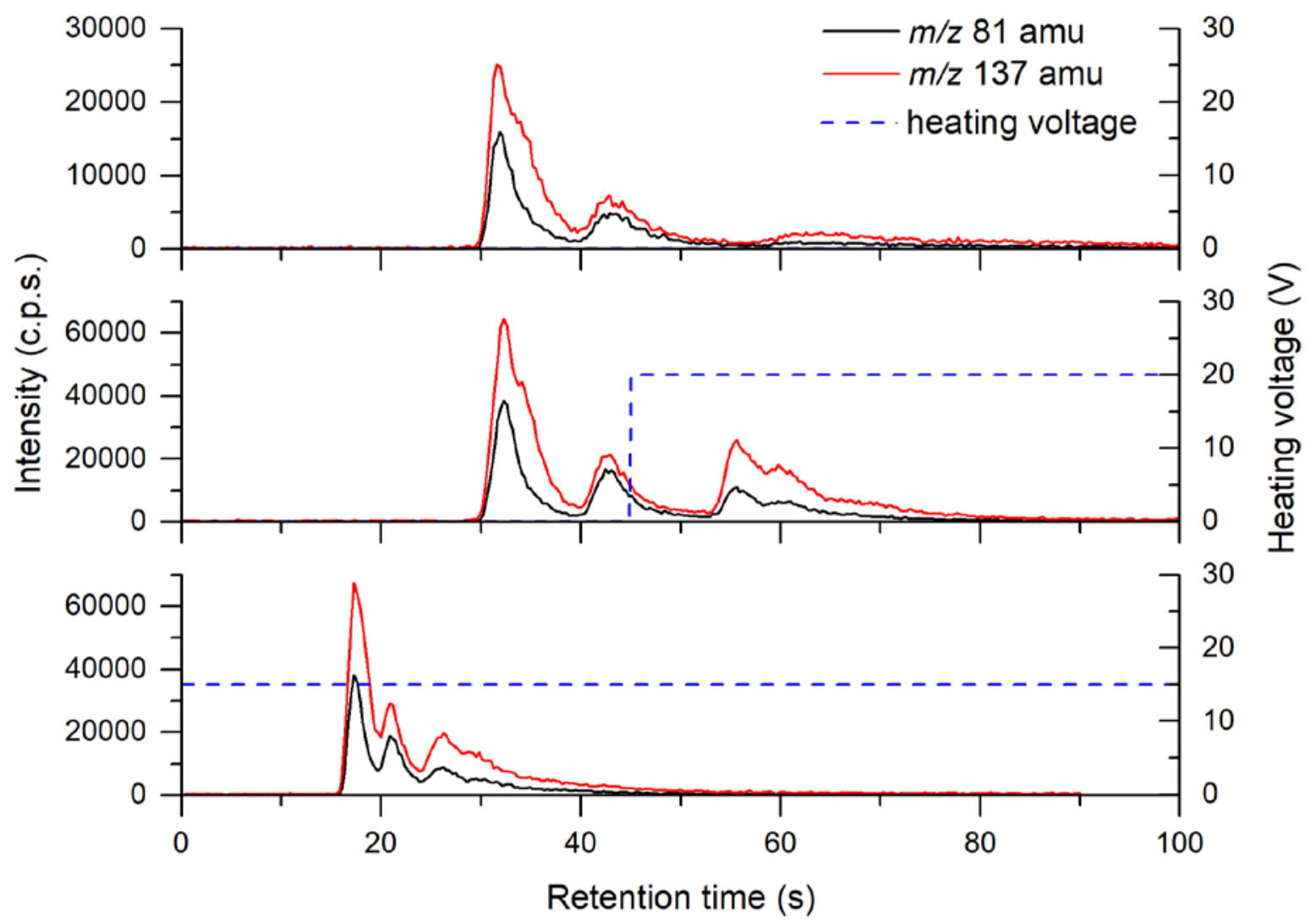

Figure S1: Chromatograms of a monoterpene mixture analysed by the MXT-1 column for different profiles of the heating voltage. Profiles were analysed by SIFT-MS using the $\mathrm{H}_{3} \mathrm{O}^{+}$reagent ion. 
Table S1: Summary of reaction rate constants and branching ratios of investigated monoterpenes. All presented rate constants have units of $10^{-9} \mathrm{~cm}^{3} \mathrm{~s}^{-1}$. Only significant products are given, for witch branching ratios are at least $10 \%$.

\begin{tabular}{|c|c|c|c|c|c|c|}
\hline & \multicolumn{2}{|l|}{$\mathrm{H}_{3} \mathrm{O}^{+}$} & \multicolumn{2}{|l|}{$\mathrm{NO}^{+}$} & \multicolumn{2}{|l|}{$\mathbf{O}_{2}^{+}$} \\
\hline & rate & Products (b.r.) & $\mathrm{k}$ & Products (b.r.) & $\mathrm{k}$ & Products (b.r.) \\
\hline$\alpha$-pinene & $\begin{array}{l}2.3^{\mathrm{a}}, \\
2.4^{\mathrm{a}^{*}}\end{array}$ & $\begin{array}{l}\mathrm{C}_{6} \mathrm{H}_{9}^{+}\left(30^{\mathrm{a}}, 39^{\mathrm{b}}\right) \\
\mathrm{C}_{10} \mathrm{H}_{17^{+}}\left(67^{\mathrm{a}}, 61^{\mathrm{b}}\right)\end{array}$ & $\begin{array}{l}2.0^{\mathrm{a}}, \\
2.0^{\mathrm{a}^{*}} \\
2.3^{\mathrm{b}}\end{array}$ & $\begin{array}{l}\mathrm{C}_{7} \mathrm{H}_{8}^{+}\left(16^{\mathrm{b}}\right) \\
\mathrm{C}_{10} \mathrm{H}_{16}{ }^{+}\left(85^{\mathrm{a}}, 77^{\mathrm{b}}\right)\end{array}$ & $\begin{array}{l}2.0^{\mathrm{a}}, \\
1.9^{\mathrm{a}^{*}} \\
2.1^{\mathrm{b}}\end{array}$ & $\begin{array}{l}\mathrm{C}_{7} \mathrm{H}_{8}{ }^{+}\left(18^{\mathrm{a}}, 22^{\mathrm{b}}\right), \\
\mathrm{C}_{7} \mathrm{H}_{9}^{+}\left(52^{\mathrm{a}}, 56^{\mathrm{b}}\right), \\
\mathrm{C}_{9} \mathrm{H}_{13}{ }^{+}\left(12^{\mathrm{a}}, 12^{\mathrm{b}}\right)\end{array}$ \\
\hline$\beta$-pinene & $\begin{array}{l}2.4^{\mathrm{a}}, \\
2.6^{\mathrm{a}^{*}}\end{array}$ & $\begin{array}{l}\mathrm{C}_{6} \mathrm{H}_{9}^{+}\left(33^{\mathrm{a}}, 40^{\mathrm{b}}\right), \\
\mathrm{C}_{10} \mathrm{H}_{17}{ }^{+}\left(64^{\mathrm{a}}, 60^{\mathrm{b}}\right)\end{array}$ & $\begin{array}{l}2.1^{\mathrm{a}} \\
2.2^{\mathrm{a}^{*}} \\
2.1^{\mathrm{b}}\end{array}$ & $\mathrm{C}_{10} \mathrm{H}_{16}{ }^{+}\left(93^{\mathrm{a}}, 89^{\mathrm{b}}\right)$ & $\begin{array}{l}2.1^{\mathrm{a}} \\
2.1^{\mathrm{a}^{*}} \\
2.0^{\mathrm{b}}\end{array}$ & $\begin{array}{l}\mathrm{C}_{7} \mathrm{H}_{9}^{+}\left(56^{\mathrm{a}}, 19^{\mathrm{b}}\right) \\
\mathrm{C}_{9} \mathrm{H}_{13^{+}}{ }^{+}\left(49^{\mathrm{b}}\right) \\
\mathrm{C}_{10} \mathrm{H}_{16^{+}}{ }^{+}\left(11^{\mathrm{a}}\right)\end{array}$ \\
\hline R-limonene & $\begin{array}{l}2.6^{\mathrm{a}} \\
2.6^{\mathrm{a}^{*}}\end{array}$ & $\begin{array}{l}\mathrm{C}_{6} \mathrm{H}_{9}^{+}\left(22^{\mathrm{a}}, 29^{\mathrm{b}}\right), \\
\mathrm{C}_{10} \mathrm{H}_{17^{+}}\left(73^{\mathrm{a}}, 68^{\mathrm{b}}\right)\end{array}$ & $\begin{array}{l}2.2^{\mathrm{a}}, \\
2.2^{\mathrm{a}^{*}} \\
2.2^{\mathrm{b}}\end{array}$ & $\mathrm{C}_{10} \mathrm{H}_{16}{ }^{+}\left(91^{\mathrm{a}}, 89^{\mathrm{b}}\right)$ & $\begin{array}{l}2.2^{\mathrm{a}} \\
2.1^{\mathrm{a}^{*}} \\
2.2^{\mathrm{b}}\end{array}$ & $\begin{array}{l}\mathrm{C}_{5} \mathrm{H}_{8}{ }^{+}\left(10^{\mathrm{b}}\right), \\
\mathrm{C}_{7} \mathrm{H}_{8}{ }^{+}\left(10^{\mathrm{b}}\right), \\
\mathrm{C}_{7} \mathrm{H}_{9}{ }^{+}\left(26^{\mathrm{a}}, 30^{\mathrm{b}}\right), \\
\mathrm{C}_{7} \mathrm{H}_{10}{ }^{+}\left(11^{\mathrm{a}}, 12^{\mathrm{b}}\right), \\
\mathrm{C}_{8} \mathrm{H}_{11^{+}}\left(11^{\mathrm{b}}\right), \\
\mathrm{C}_{9} \mathrm{H}_{13}{ }^{+}\left(14^{\mathrm{a}}, 13^{\mathrm{b}}\right), \\
\mathrm{C}_{10} \mathrm{H}_{16}{ }^{+}\left(11^{\mathrm{a}}, 11^{\mathrm{b}}\right)\end{array}$ \\
\hline 3-carene & $\begin{array}{l}2.3^{\mathrm{a}} \\
2.4^{\mathrm{a}^{*}}\end{array}$ & $\begin{array}{l}\mathrm{C}_{6} \mathrm{H}_{9}^{+}\left(19^{\mathrm{a}}, 24^{\mathrm{b}}\right), \\
\mathrm{C}_{10} \mathrm{H}_{17^{+}}\left(78^{\mathrm{a}}, 76^{\mathrm{b}}\right)\end{array}$ & $\begin{array}{l}2.1^{\mathrm{a}}, \\
2.0^{\mathrm{a}^{*}} \\
2.2^{\mathrm{b}}\end{array}$ & $\mathrm{C}_{10} \mathrm{H}_{16}{ }^{+}\left(86^{\mathrm{a}}, 81^{\mathrm{b}}\right)$ & $\begin{array}{l}2.0^{\mathrm{a}} \\
2.0^{\mathrm{a}^{*}} \\
1.9^{\mathrm{b}}\end{array}$ & $\begin{array}{l}\mathrm{C}_{7} \mathrm{H}_{8}^{+}\left(11^{\mathrm{b}}\right), \\
\mathrm{C}_{7} \mathrm{H}_{9}^{+}\left(41^{\mathrm{a}}, 45^{\mathrm{b}}\right), \\
\mathrm{C}_{9} \mathrm{H}_{13}{ }^{+}\left(20^{\mathrm{a}}, 20^{\mathrm{b}}\right), \\
\mathrm{C}_{10} \mathrm{H}_{16^{+}}\left(14^{\mathrm{a}}\right)\end{array}$ \\
\hline myrcene & $\begin{array}{l}2.6^{\mathrm{a}}, \\
2.7^{\mathrm{*}}\end{array}$ & $\begin{array}{l}\mathrm{C}_{6} \mathrm{H}_{9}^{+}\left(26^{\mathrm{a}}, 30^{\mathrm{b}}\right), \\
\mathrm{C}_{10} \mathrm{H}_{17}{ }^{+}\left(59^{\mathrm{a}}, 58^{\mathrm{b}}\right)\end{array}$ & $\begin{array}{l}2.3^{\mathrm{a}}, \\
2.2^{\mathrm{a}^{*}} \\
2.2^{\mathrm{b}}\end{array}$ & $\begin{array}{l}\mathrm{C}_{7} \mathrm{H}_{8}^{+}\left(11^{\mathrm{b}}\right), \\
\mathrm{C}_{7} \mathrm{H}_{9}^{+}\left(22^{\mathrm{a}}, 34^{\mathrm{b}}\right) \\
\mathrm{C}_{10} \mathrm{H}_{16^{+}}\left(61^{\mathrm{a}}, 55^{\mathrm{b}}\right)\end{array}$ & $\begin{array}{l}2.2^{\mathrm{a}}, \\
2.2^{\mathrm{a}^{*}} \\
2.2^{\mathrm{b}}\end{array}$ & $\begin{array}{l}\mathrm{C}_{5} \mathrm{H}_{9}^{+}\left(10^{\mathrm{b}}\right) \\
\mathrm{C}_{7} \mathrm{H}_{8}^{+}\left(70^{\mathrm{b}}\right) \\
\mathrm{C}_{7} \mathrm{H}_{9}^{+}\left(61^{\mathrm{a}}\right)\end{array}$ \\
\hline camphene & $\begin{array}{l}2.4^{\mathrm{a}}, \\
2.6^{\mathrm{a}^{*}}\end{array}$ & $\begin{array}{l}\mathrm{C}_{6} \mathrm{H}_{9}^{+}\left(14^{\mathrm{b}}\right), \\
\mathrm{C}_{10} \mathrm{H}_{17}{ }^{+}\left(88^{\mathrm{a}}, 86^{\mathrm{b}}\right)\end{array}$ & $\begin{array}{l}2.1^{\mathrm{a}}, \\
2.1^{\mathrm{a}^{*}} \\
2.3^{\mathrm{b}}\end{array}$ & $\begin{array}{l}\mathrm{C}_{10} \mathrm{H}_{16}{ }^{+}\left(87^{\mathrm{a}}, 79^{\mathrm{b}}\right) \\
\mathrm{NO}^{+} \mathrm{C}_{10} \mathrm{H}_{16}\left(11^{\mathrm{b}}\right)\end{array}$ & $\begin{array}{l}2.0^{\mathrm{a}}, \\
2.1^{\mathrm{a}^{*}} \\
2.2^{\mathrm{b}}\end{array}$ & $\begin{array}{l}\mathrm{C}_{7} \mathrm{H}_{9}{ }^{+}\left(13^{\mathrm{a}}, 19^{\mathrm{b}}\right), \\
\mathrm{C}_{8} \mathrm{H}_{11^{+}}\left(10^{\mathrm{b}}\right), \\
\mathrm{C}_{9} \mathrm{H}_{13}{ }^{+}\left(44^{\mathrm{a}}, 49^{\mathrm{b}}\right)\end{array}$ \\
\hline$\alpha$-terpinene & & $\begin{array}{l}\mathrm{C}_{6} \mathrm{H}_{9}^{+}\left(10^{\mathrm{b}}\right), \\
\mathrm{C}_{10} \mathrm{H}_{17^{+}}\left(87^{\mathrm{b}}\right)\end{array}$ & $2.0^{\mathrm{b}}$ & $\mathrm{C}_{10} \mathrm{H}_{16}{ }^{+}\left(87^{\mathrm{a}}, 99^{\mathrm{b}}\right)$, & $2.0^{\mathrm{b}}$ & $\begin{array}{l}\mathrm{C}_{7} \mathrm{H}_{9}^{+}\left(16^{\mathrm{b}}\right) \\
\mathrm{C}_{9} \mathrm{H}_{13}{ }^{+}\left(42^{\mathrm{b}}\right) \\
\mathrm{C}_{10} \mathrm{H}_{16}^{+}\left(33^{\mathrm{b}}\right)\end{array}$ \\
\hline$\gamma$-terpinene & & $\begin{array}{l}\mathrm{C}_{6} \mathrm{H}_{9}^{+}\left(17^{\mathrm{b}}\right) \\
\mathrm{C}_{10} \mathrm{H}_{17^{+}}\left(81^{\mathrm{b}}\right)\end{array}$ & $2.1^{\mathrm{b}}$ & $\begin{array}{l}\mathrm{C}_{10} \mathrm{H}_{15^{+}}{ }^{+}\left(18^{\mathrm{b}}\right), \\
\mathrm{C}_{10} \mathrm{H}_{16^{+}}\left(87^{\mathrm{a}}, 75^{\mathrm{b}}\right),\end{array}$ & $1.9^{\mathrm{b}}$ & $\begin{array}{l}\mathrm{C}_{7} \mathrm{H}_{8}^{+}\left(12^{\mathrm{b}}\right), \\
\mathrm{C}_{7} \mathrm{H}_{9}^{+}\left(46^{\mathrm{b}}\right), \\
\mathrm{C}_{9} \mathrm{H}_{13}{ }^{+}\left(21^{\mathrm{b}}\right), \\
\mathrm{C}_{10} \mathrm{H}_{16^{+}}\left(14^{\mathrm{b}}\right)\end{array}$ \\
\hline
\end{tabular}

${ }^{\mathrm{a}}$ (Schoon et al., 2003); ${ }^{\mathrm{b}}$ (Wang et al., 2003); ${ }^{\mathrm{c}}$ Present result based on SIFT-MS measurements; ${ }^{\mathrm{d}}$ Present result based on fastGC-SIFT-MS measurements; * theoretical data based on the method of Su and Chesnavitch (Su and Chesnavich, 1982); b.r. stands for branching ratio; Dimension of rate constants is $10^{-9} \mathrm{~cm}^{3} \mathrm{~s}^{-1}$. 

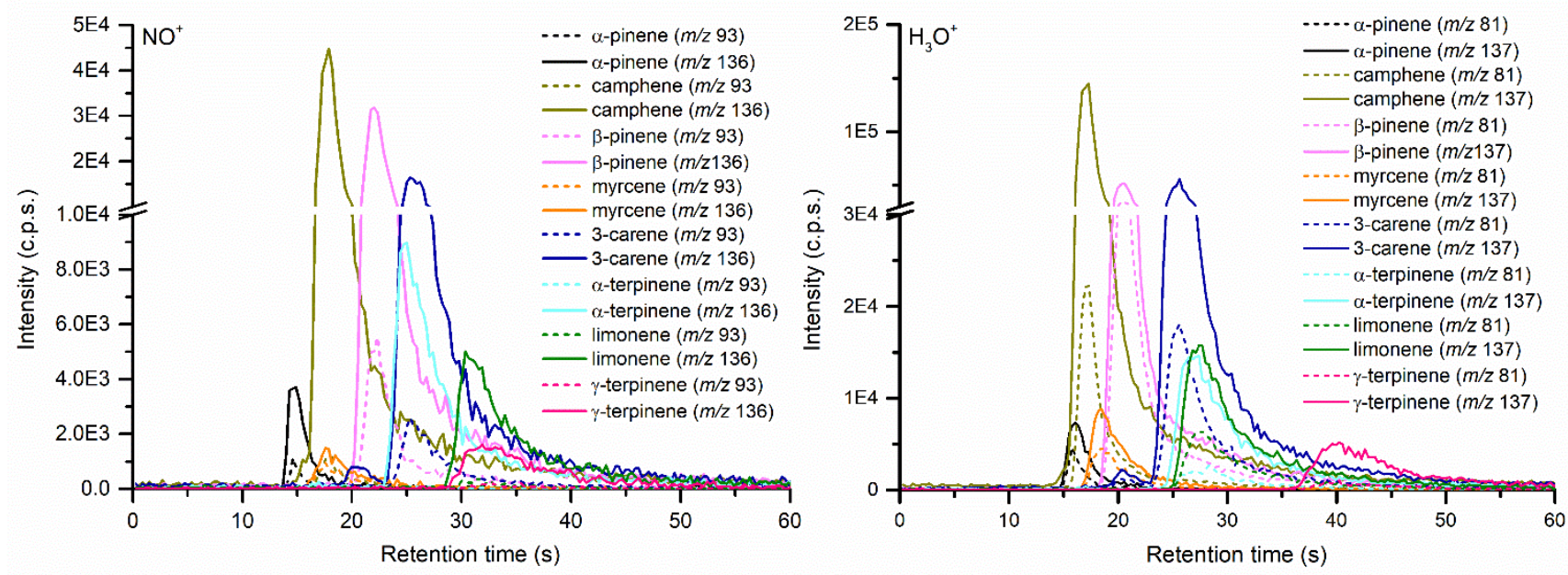

Figure S2: Chromatograms of individual monoterpenes analysed using the MXT-1 column at a constant temperature of column $\sim 40^{\circ} \mathrm{C}$. The profile is associated with the profile shown in the bottom of Figure S1. Profiles were analysed by SIFT-MS using the $\mathrm{H}_{3} \mathrm{O}^{+}$reagent ion. Intensity of $\alpha-$ pinene was reduced. 

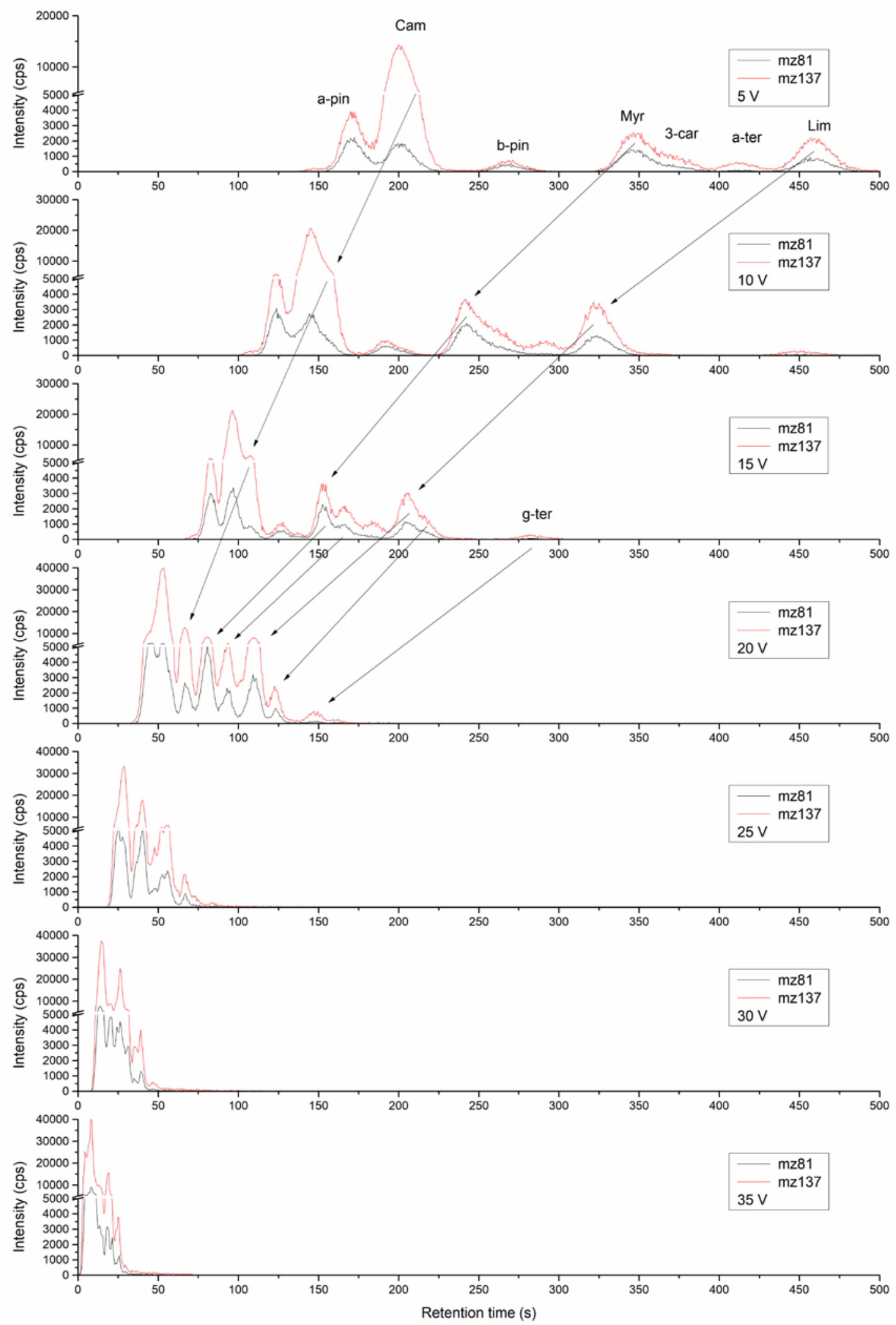

Figure S3: Chromatograms of a monoterpene mixture analysed by the MXT- Volatiles column for different heating voltages. Profiles were analysed by SIFT-MS using the $\mathrm{H}_{3} \mathrm{O}^{+}$reagent ion. 


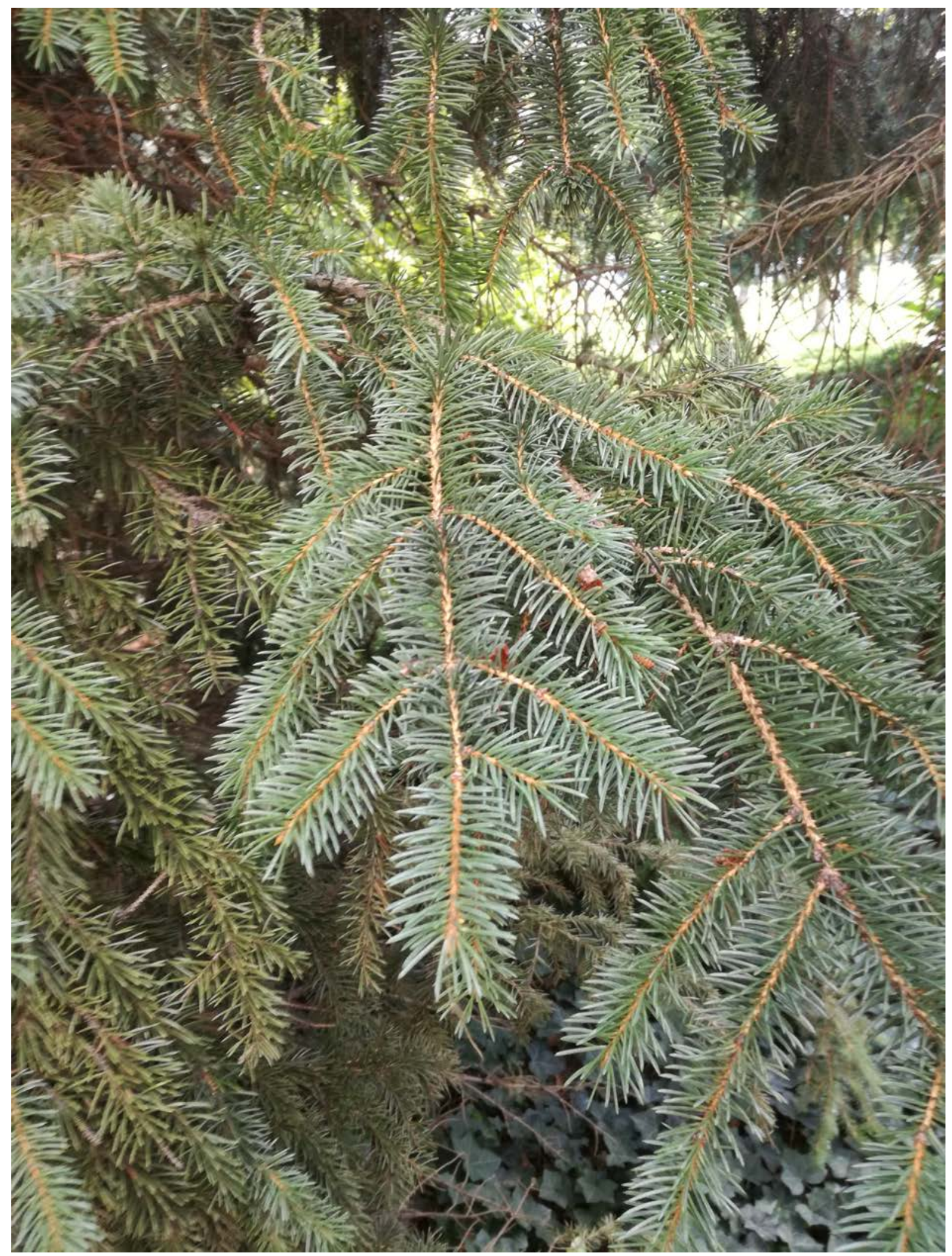

Figure S4: Sample no. 1 (Pincea punges) 


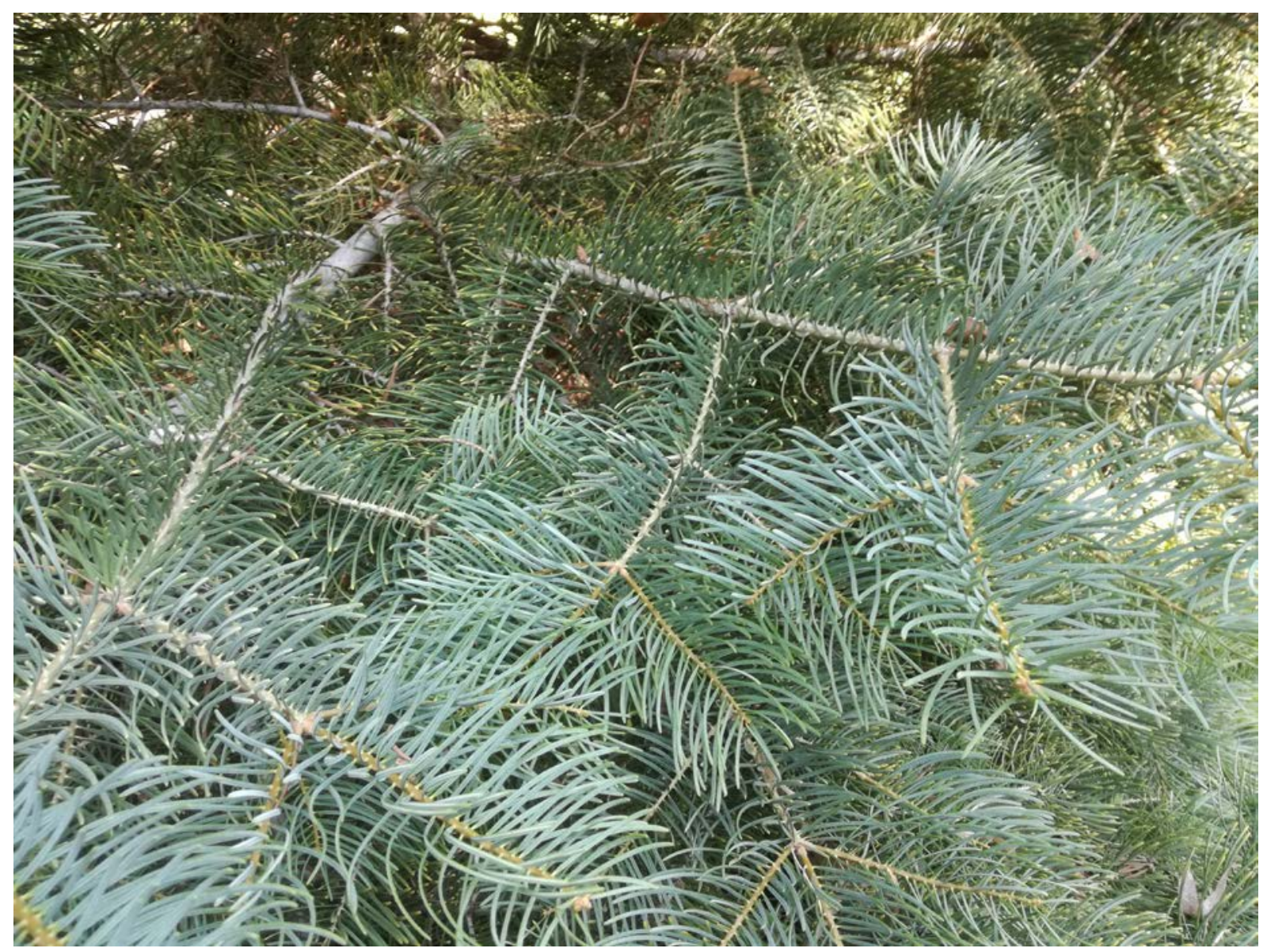

Figure S5: Sample no. 2 (Abies concolor) 


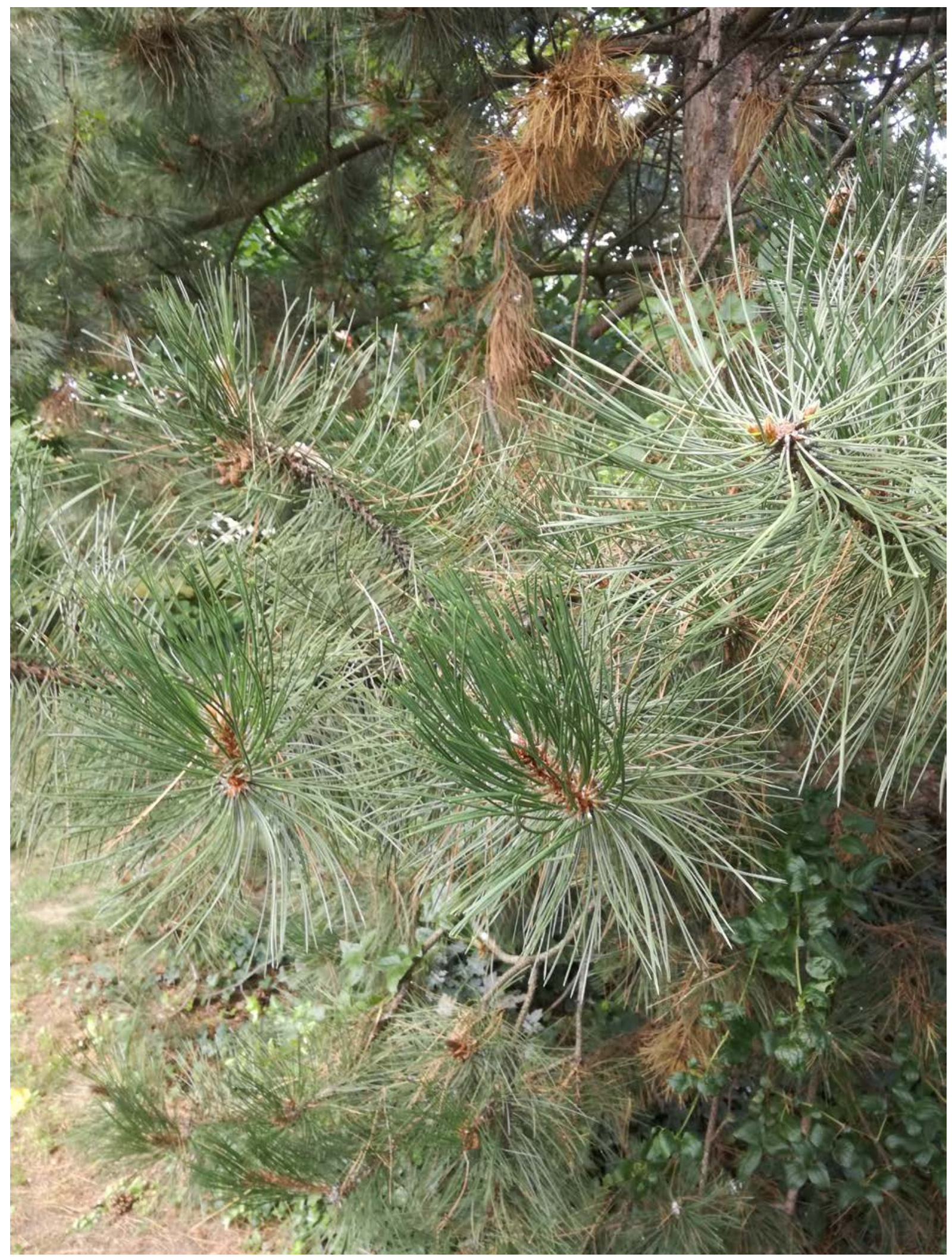

Figure S6: Sample no. 3 (Pinus nigra) 


\section{References}

Schoon, N., Amelynck, C., Vereecken, L., and Arijs, E.: A selected ion flow tube study of the reactions of $\mathrm{H}_{3} \mathrm{O}^{+}$, $\mathrm{NO}^{+}$and $\mathrm{O}_{2}{ }^{+}$with a series of monoterpenes, International Journal of Mass Spectrometry, 229, 231-240, 2003.

Su, T., and Chesnavich, W. J.: Parametrization of the ion-polar molecule collision rate constant by trajectory calculations, The Journal of Chemical Physics, 76, 5183-5185, 1982.

Wang, T., Španěl, P., and Smith, D.: Selected ion flow tube, SIFT, studies of the reactions of $\mathrm{H}_{3} \mathrm{O}^{+}$, $\mathrm{NO}^{+}$and $\mathrm{O}_{2}{ }^{+}$ with eleven $\mathrm{C}_{10} \mathrm{H}_{16}$ monoterpenes, Int. J. Mass Spec., 228, 117-126, 2003. 